\title{
Paradoxical sphincter contraction is rarely indicative of anismus
}

\author{
W A Voderholzer, D A Neuhaus, A G Klauser, K Tzavella, S A Müller-Lissner, \\ N E Schindlbeck
}

\begin{abstract}
Background-Anismus is thought to be a cause of chronic constipation by producing outlet obstruction. The underlying mechanism is paradoxical contraction of the anal sphincter or puborectalis muscle. However, paradoxical sphincter contraction (PSC) also occurs in healthy controls, so anismus may be diagnosed too often because it may be based on a non-specific finding related to untoward conditions during the anorectal examination.

Aims-To investigate the pathophysiological importance of PSC found at anorectal manometry in constipated patients and in patients with stool incontinence.

Methods-Digital rectal examination and anorectal manometry were performed in 102 chronically constipated patients, 102 patients with stool incontinence, and in 18 controls without anorectal disease. In 120 of the 222 subjects defaecography was also performed. Paradoxical sphincter contraction was defined as a sustained increase in sphincter pressure during straining. Anismus was assumed when PSC was present on anorectal manometry and digital rectal examination and the anorectal angle did not widen on defaecography.
\end{abstract}

Results-Manometric PSC occurred about twice as often in constipated patients as in incontinent patients $(41.2 \%$ versus $25.5 \%, p<0.017)$ and its prevalence was similar in incontinent patients and controls $(25.5 \%$ versus $22.2 \%)$. Oroanal or rectosigmoid transit times in constipated patients with and without PSC did not differ significantly (total 64.6 (8.9) hours versus $54.2(8.1)$ hours; rectosigmoid 14.9 (2.4) hours versus 13.8 (2.5) hours). Conclusions-Paradoxical sphincter contraction is a common finding in healthy controls as well as in patients with chronic constipation and stool incontinence. Hence, PSC is primarily a laboratory artefact and true anismus is rare. (Gut 1997; 41: 258-262)

Keywords: anismus; paradoxical sphincter contraction; constipation; stool incontinence; anorectal manometry

Correspondence to: Dr W A Voderholzer, Medizinische Klinik, Klinikum Innenstadt, Universität München, Ziemssenstrasse 1, D-80336 München, Germany.

Accepted for publication 10 March 1997
Anismus is thought to cause constipation by producing outlet obstruction during defaecation. It has been described especially in young or middle aged women with chronic constipation $^{12}$ and may be associated with the inability to defaecate a water-filled rectal balloon. Its main feature is an inappropriate contraction (paradoxical sphincter contraction, PSC) rather than relaxation of the anal sphincter or puborectalis muscle during straining.

Paradoxical sphincter relaxation may be shown by digital rectal examination, manometry, electromyographic examination, and-as a failure of the anorectal angle to widen-on defaecography. As PSC is also found in control subjects without anorectal disease ${ }^{3}$ it may be a non-specific finding related to untoward conditions during examination. It seems natural that an embarrassed patient does not show normal defaecation behaviour. Being afraid of losing stools may play an important part in PSC during straining.

The aim of this study was therefore to find out the pathophysiological importance of the anorectal manometric finding "paradoxical sphincter contraction during straining".

\section{Methods}

One hundred and two consecutive constipated patients and 102 consecutive patients with stool incontinence were included from our gastroenterological outpatient department. Patients were considered to be constipated if they had had fewer than three bowel movements per week and/or a frequent ( $>25 \%$ ) need to strain during defaecation for more than one year and who presented to the hospital because of constipation. Organic disease of the colon had been excluded by colonoscopy, laboratory findings (TSH), and further investigations by the general practitioner. Stool incontinence was defined as an unintentional loss of faeces at least once a week for more than six months.

Eighteen patients referred for colonic surgery or for colonoscopy to exclude colonic malignancy, who were completely free of symptoms, acted as controls.

\section{ANORECTAL MANOMETRY}

Anorectal manometry was performed using a seven-lumen side hole catheter (Arndorfer, Greendale, Wisconsin, USA) which was continuously perfused with distilled water at a rate of $0.1 \mathrm{ml} / \mathrm{min}$ per recording site (fluid perfusion device, Mui, Mississauga, Canada). There were six side holes, $0.5 \mathrm{~cm}$ apart at about $15 \mathrm{~cm}$ from the tip of the catheter, radially orientated for examination of the anorectal sphincter muscle, and another side hole was situated $5 \mathrm{~cm}$ from the tip of the catheter for determination of the rectal pressure. At the very tip of the catheter a latex balloon was 
TABLE 1 Mean (SEM) demographic and manometric data of pathophysiological groups studied. Results of transit time measurements are mentioned in the text and figure 1

\begin{tabular}{llll}
\hline & Controls & $\begin{array}{l}\text { Chronic } \\
\text { constipation }\end{array}$ & Stool incontinence \\
\hline Sex ratio (female/male) & $10 / 8$ & $94 / 8^{\star}$ & $89 / 13^{\star}$ \\
Age (years) & $49.1(3.3)$ & $52.9(1.6)$ & $61.2(1.3)^{\star} \dagger$ \\
Symptom duration (years) & - & $20.5(1.6)$ & $5.2(1.1)^{\circ}$ \\
Sphincter pressures: & $62.1(4.5)$ & $50.8(1.8)^{\star}$ & $32.9(1.8)^{\star} \dagger$ \\
$\quad$ Resting (mm Hg) & $102.6(6.5)$ & $89.7(2.8)^{\star}$ & $62.9(3.8)^{\star} \dagger$ \\
$\quad$ Maximal (mm Hg) & $20.0(0.8)$ & $21.2(1.1)$ & $22.1(1.0)$ \\
RAIR thresholds: & $85.3(5.4)$ & $85.0(3.5)$ & $78.2(4.1)$ \\
$\quad$ First appearance (ml) & $22.2(1.5)$ & $28.9(2.0)$ & $28.2(1.6)$ \\
$\quad$ Maximal relaxation (ml) & $44.7(3.4)$ & $74.7(4.1)^{\star}$ & $61.3(3.4)$ \\
Rectal sensitivity thresholds: & $4(22.2)$ & $42(41.2)$ & $26(25.5) \ddagger$ \\
$\quad \begin{array}{l}\text { Perception (ml) } \\
\text { Urge (ml) }\end{array}$ & &
\end{tabular}

PSC, paradoxical sphincter contraction; RAIR, rectoanal inhibitory reflex.

${ }^{\star} \mathrm{p}<0.05$ versus controls, $\mathrm{\dagger p}<0.05$ versus constipation, $\neq \mathrm{p}=0.017$ versus constipation.

TABLE 2 Mean (SD) demographic and manometric data of patients with and without manometric paradoxical sphincter contraction (PSC). The results did not change when groups were stratified according to constipation, stool incontinence and controls

\begin{tabular}{lll}
\hline & PSC (n=72) & No PSC (n=150) \\
\hline Sex ratio (female/male) & $58 / 14$ & $135 / 15$ \\
Age (years) & $55.7(2.0)$ & $56.7(1.2)$ \\
Symptom duration & $12.7(1.6)$ & $14.5(1.7)$ \\
Sphincter pressures: & $45.8(2.8)$ & $42.4(1.6)$ \\
$\quad$ Resting pressure (mm Hg) & $81.7(4.3)$ & $76.1(3.0)$ \\
$\quad$ Maximal squeeze pressure (mm Hg) & & \\
RAIR thresholds: & $21.5(1.4)$ & $21.5(0.8)$ \\
$\quad$ First appearance (ml) & $83.8(4.9)$ & $81.5(2.9)$ \\
$\quad$ Maximal relaxation (ml) & & \\
Rectal sensitivity thresholds: & $29.2(2.3)$ & $27.4(1.4)$ \\
$\quad$ Perception (ml) & $69.4(5.0)$ & $64.4(2.9)$ \\
\hline Urge (ml) & &
\end{tabular}

RAIR, rectoanal inhibitory reflex.

attached which could be blown up by a syringe to a volume of $150 \mathrm{ml}$.

Examination started with a rectal digital examination with the patient lying on the left side. During digital examination the sphincter pressure and reaction of the sphincter muscle and pelvic floor during straining and squeezing were determined. The manometry tube was then introduced into the rectum up to about $20 \mathrm{~cm}$ from the dentate line and slowly withdrawn until a rise in pressure indicated the position of the anorectal sphincter muscle. The length of the anal high pressure zone was then determined by slowly pulling back the manometry tube stepwise by $1 \mathrm{~cm}$. The patient was asked to squeeze and strain three times. The balloon on the tip of the catheter was blown up stepwise $(10 \mathrm{ml}$ per step) and the balloon volume at which the patient experienced sensation and urge to defaecate was determined by asking the patient what he or she felt. Any pain during balloon distension was also recorded.

Paradoxical sphincter contraction was defined as any sustained rise in anal pressure during straining without consecutive relaxation of the anal sphincter muscle.

DEFAECOGRAPHY AND MEASUREMENT OF TRANSIT TIME

In 94 patients $(42.3 \%)$ defaecography was performed as described previously. ${ }^{4}$ Briefly, $150 \mathrm{ml}$ of viscous radio-opaque contrast medium was instilled into the rectum via a large bore tube. The patient was then seated on a plastic toilet in front of an $x$-ray camera and 10-30 pictures (100 mm camera, $110 \mathrm{kV}$ ) were taken from the side while the patient was

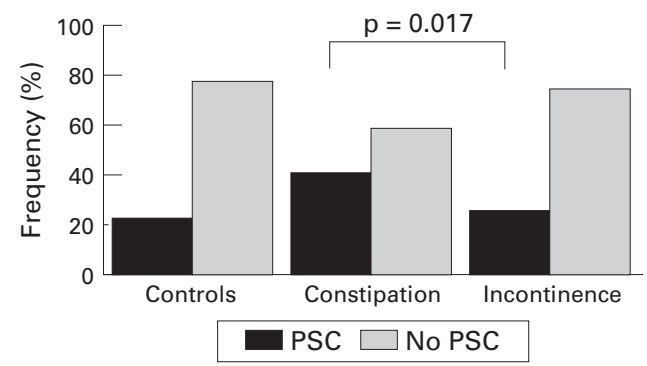

Figure 1: Frequency of manometric paradoxical sphincter contraction (PSC) in the three groups studied. The ordinate shows the percentage of patients in each group with and without PSC. If the data from the controls and incontinent patients were pooled the $p$ value decreased $(p=0.01)$.

asked to empty his or her bowel as completely as possible. Paradoxical sphincter contraction was defined as a failure to widen the anorectal angle and anal canal during straining accompanied by a persistent dorsal indentation at the upper border of the anal canal and remnants of rectal contrast medium after defaecation indicating grossly delayed rectal emptying.

Transit time was measured in $62(60.8 \%)$ constipated patients using the radio-opaque marker method. Briefly, 20 radio-opaque encapsulated pellets were ingested for 14 days at 0900 hours and one day after the last capsule was taken an abdominal $x$-ray was performed at 0900 hours. The number of pellets on the abdominal film was counted and multiplied by 1.2 to give the oroanal transit time. ${ }^{5}$ For determination of segmental colonic transit time lines were drawn on the abdominal film as described previously ${ }^{6}$ and defined as right colon, left colon, and rectosigmoid colon.

\section{STATISTICS}

The Mann-Whitney $U$ test was used to compare numerical data of the different groups and the $\chi^{2}$ test was used to compare nominal data. A $\mathrm{p}$ value of $<0.05$ was considered significant.

\section{Results}

DEMOGRAPHIC DATA

One hundred and two constipated patients, 102 incontinent patients, and 18 healthy controls were studied. The incontinent patients were older than the constipated patients and control subjects, while symptom duration was longer in the constipated patients than in the incontinent patients. There were more women in the patient groups than in the control group, but there was no difference with respect to age and sex distribution between the constipated and incontinent patients (table 1). When patients with and without PSC were compared no differences in demographic data were found (table 2).

\section{ANORECTAL MANOMETRY}

Incontinent patients presented with lower sphincter resting and maximal squeeze pressures than controls and constipated patients. In addition, sphincter pressures were lower in constipated patients than in controls (table 1). Thresholds for first appearance or maximum relaxation of the rectoanal inhibitory reflex did 


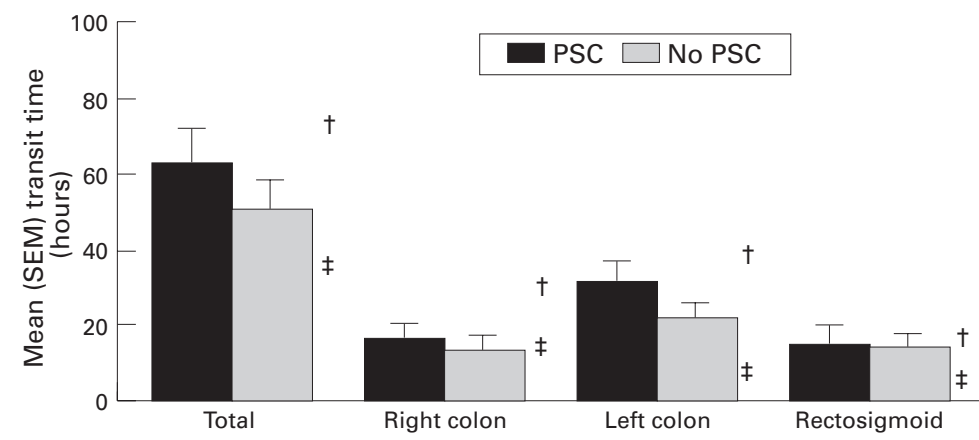

Figure 2: Mean (SEM) total and segmental transit times in constipated patients with ( $n$ $=29)$ and without $(n=33)$ PSC. + and $\ddagger$ represent two patients in whom manometric, defaecographic and proctographic examinations revealed PSC, thus establishing the diagnosis of "anismus". Note that † had slow total transit as well as slow segmental (right and left hemicolon) transit.
The frequency of PSC on manometry, defaecography, and rectal digital examination is shown in fig 3. There were only three patients in whom all three examinations confirmed PSC. All were constipated patients who complained of a low stool frequency and the necessity to strain at stools. Transit times were obtained for two of these patients (fig 2). One patient had a slow total transit time (72.0 hours) and slow segmental transit times in the right (28.8 hours) and left (38.4 hours) colon, but a normal transit time in the rectosigmoid colon (13.2 hours). The second patient had a normal total transit time (34.8 hours) and segmental transit times of 16.8 hours, 4.8 hours, and 4.8 hours for the right, left, and rectosigmoid colon, respectively.

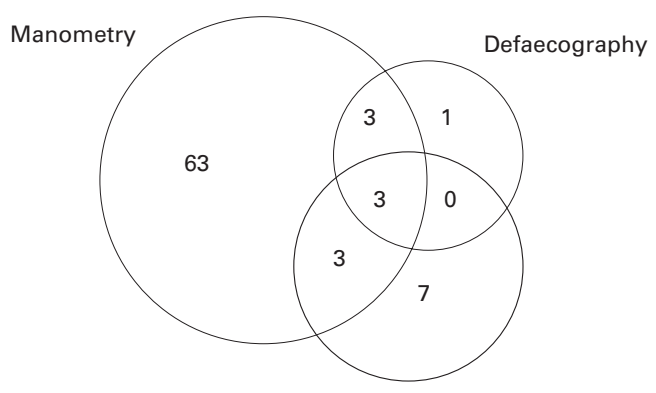

Digital examination

Figure 3: Frequency of examinations that proved positive for PSC in patients with defaecography, manometry, and digital rectal examination.

not differ between the three groups studied. Compared with controls, constipated patients had higher rectal sensitivity thresholds for the urge to defaecate (table 1).

Paradoxical sphincter contraction was present in $22.2 \%$ of control subjects, $41.2 \%$ of constipated patients, and $25.5 \%$ of incontinent patients. Although PSC occurred more frequently in constipated patients than in control subjects, the difference was not statistically significant ( $\mathrm{p}=0.14, \chi^{2}$ test). However, PSC was more common in constipated patients than in incontinent patients $(p=0.017$, fig 1$)$ or than in control subjects and incontinent patients combined $(\mathrm{p}=0.01)$.

\section{TRANSIT TIMES}

Total and segmental transit times were measured in constipated patients only. The mean (SEM) total transit time was 55.5 (5.9) hours, and segmental transit times were 15.0 (1.8) hours, 26.2 (3.3) hours, and 14.3 (1.7) hours in the right, left, and rectosigmoid colon, respectively. Transit times did not differ when patients were stratified according to the presence of PSC (fig 2).

\section{PARADOXICAL SPHINCTER CONTRACTION IN} COMBINED EXAMINATIONS

Manometry and rectal digital examination were performed in all patients and PSC was found in $72(32.4 \%)$ and $13(5.8 \%)$ subjects, respectively. Defaecography, which was performed in 94 patients, revealed PSC in seven $(7.4 \%)$ patients.

\section{Discussion}

Anismus or "spastic pelvic floor syndrome" is thought to be one cause of severe constipation. ${ }^{2}$ Its pathophysiological basis is PSC during straining. However, there is no single test by which the diagnosis can be confirmed so it is possible that it is a laboratory artefact. None the less, operations to solve the problem by posterior division of the puborectalis muscle have been performed. ${ }^{8}$

During the past few years functional outlet obstruction has been widely acknowledged as a cause of chronic constipation. In organic diseases such as tumour stenosis, outlet rectal tumour mass. On the other hand, in functional forms of outlet obstruction the physiology is normal when the patient does not defaecate and becomes pathological only traction during straining has been claimed as one of the main causes of functional outlet obstruction. ${ }^{9}$

The concept of PSC as a specific finding in constipated patients has been questioned in several studies. Jones et al found PSC of the puborectalis muscle in $76 \%$ of constipated patients, and also in $48 \%$ of patients with idiopathic perineal pain and in 50\% with solitary rectal ulcer syndrome. ${ }^{10}$ Another study found maintenance of the positive anorectal pressure gradient during defaecation (which may be interpreted as an equivalent of PSC) in patients with suspected outlet obstruction. However, $80 \%$ of these patients showed appropriate sphincter relaxation when manometry was performed at home indicating that PSC in the laboratory setting may be an artefact. ${ }^{1} \mathrm{Up}$ have anismus by Barnes and Lennard-Jones, ${ }^{3}$ and it has also been reported in up to $50 \%$ of patients with stool incontinence ${ }^{1112}$ which also may not fit into the concept of PSC being the cause of constipation.

On the other hand, Kuijpers et al found rectal marker delay and/or abnormal marker distribution in 22 of 24 patients with radiologically diagnosed PSC, ${ }^{7}$ suggesting the functional importance of PSC. However, to date no other study has shown this. In 132 patients with slow transit constipation, of whom $50 \%$ exhibited anismus as defined by defaecoobstruction is continuously maintained by a during defaecation. Paradoxical sphincter conto one third of normal subjects were found to 
TABLE 3 Findings on defaecography in 94 patients

\begin{tabular}{ll}
\hline Finding & Frequency (\%) \\
\hline No pathological finding & $12(12.8)$ \\
PSC alone & $3(3.2)$ \\
PSC and rectocele & $4(4.2)$ \\
Rectocele alone & $9(9.6)$ \\
Rectocele and internal rectal prolapse & $23(24.4)$ \\
Rectocele and others (except anismus) & $15(16.0)$ \\
Internal rectal prolapse alone & $19(20.2)$ \\
Others & $9(9.6)$ \\
Total & $94(100)$ \\
\hline
\end{tabular}

PSC $=$ paradoxical sphincter contraction.

graphy, no correlation was found between the retention of markers in the rectosigmoid segment and outlet obstruction. ${ }^{13}$ Correspondingly, another study could not detect stasis of rectal markers in patients with and without PSC identified by proctography. ${ }^{14}$ There is also evidence from biofeedback studies that measurement of colonic transit time is a less specific test for confirmation of the diagnosis of "anismus". ${ }^{15}$ Our data support the hypothesis of PSC being a non-specific finding rather than a disease entity since PSC was also found in a considerable percentage of control subjects and, in addition, total and segmental transit times were not increased in patients with PSC. In particular, there was no evidence for a delay in the transit of rectal markers in these patients (table 2).

How can the discrepancies with the findings of Kuijpers et al be explained? Kuijpers et al used a different method for measuring transit time by applying a single marker dose and performing multiple $x$-rays. In 10 of 22 patients the abnormal distribution of rectal markers was seen only on days 1 and 2 after marker ingestion whereas rectal emptying on day 4 was even faster than in controls. Moreover, total colonic transit times were increased in the majority of patients, signifying slow transit in more than one segment of the colon. Pure rectal stasis (as the main cause of prolonged total transit) could be confirmed in five patients only, so PSC cannot be dismissed as an artefact in at least some of their patients.

One possible drawback of the study by Kuijpers et al is the fact that the diagnosis was established by only one type of examination (radiology). A finding is unlikely to be an artefact if it is found by several different examinations. Our results show that the number of patients fell rapidly when two or more PSC positive examinations were taken to establish the diagnosis of "anismus" (fig 3). It is suggested that all three of the following examinations should show PSC in order to diagnose "anismus": anorectal manometry, defaecography, rectal digital examination.

It is interesting to note that in our study both incontinent and constipated patients had lower resting and maximal sphincter pressures than controls (table 1). The mean age difference between constipated patients and controls was only about four years, so age may not be the only factor in the lower sphincter pressures of these patients. Our data support the concept of neuropathic sphincter weakness due to exces- sive straining over many years in constipated patients.

In our study three constipated patients presented with anismus by manometry, digital examination, and defaecography. In two of them transit times were measured; one was found to have a slow transit time and the other had normal transit values. From these and other results it seems that patients with anismus may be a heterogenous group with both pathological and normal colonic transit. How can this be explained? In a previous study we found that suppression of the urge to defaecate can lead to a prolonged transit time, not only in the rectum but also in the right hemicolon. ${ }^{16}$ Interpretation of our results, however, suggests that this condition is rare. Slow transit may occur in patients with PSC if there is a functional obstruction resulting in a low stool frequency. On the other hand, passage through the colon may remain normal in spite of PSC if the patient is able to overcome anorectal obstruction by severe straining. Nevertheless, all patients with anismus may suffer from symptoms of disordered defaecation due to PSC resulting in the necessity to strain at stools. This may also explain why PSC occurs more often in patients with constipation than in those with other forms of anorectal disease $\mathrm{e}^{10}$ and is in accordance with other studies that have reported evidence of defaecatory disorders in patients with chronic constipation compared with controls. ${ }^{17}{ }^{18}$ This is confirmed by our data showing that about twice as many constipated patients presented with PSC compared with patients with stool incontinence and controls (table 1).

From this, however, it may also be concluded that PSC is the consequence rather than the cause of constipation. Straining at stools is one of the main symptoms of constipated patients. Patients normally have to strain in order to empty small and hard stools. Frequent and severe attempts to strain may lead to coinnervation of the external anal sphincter and/or the puborectalis muscle. If the patient is not aware of this he or she may establish incorrect defaecation behaviour. Evidence for this is given by the positive results of biofeedback training in the majority of patients with PSC. ${ }^{15}$ Removal of the cause for straining may therefore resolve the problem of anismus. ${ }^{19}$

It should also be noted that both examination under laboratory conditions and evacuation of contrast media in the $x$-ray department are based on the assumption that voluntary straining reflects natural defaecation behaviour. However, this may only be the case when a desire to defaecate occurs along with coordinated combination of abdominal muscle contraction and pelvic floor and anal sphincter relaxation. It is easy to understand that these assumptions may not apply to examinations under artificial conditions. Voluntary straining may therefore produce a Valsalva response resulting in sphincter contraction rather than normal defaecation behaviour.

In conclusion, paradoxical sphincter contraction is a common finding in healthy controls as well as in patients with chronic con- 
stipation and stool incontinence. Our data therefore support the hypothesis that PSC is primarily a laboratory artefact. However, since there is a trend towards a higher frequency of PSC in patients with chronic constipation, there may be a subgroup of constipated patients who suffer from anismus as their main functional disorder.

This study was supported by Deutsche Forschungsgemeinschaft grant Kl 641/4-1.

1 Duthie GS, Bartolo DCC. Anismus: the cause of constipation? Results of investigation and treatment. World $\mathcal{F}$ Surg 1992; 16: 831-5.

2 Preston DM, Lennard Jones JE. Anismus in chronic constipation. Dig Dis Sci 1985; 30: 413-8.

3 Barnes PR, Lennard-Jones JE. Function of the striated anal sphincter during straining in control subjects and constiidiopathic megacolon. Int 7 Colorectal Dis 1988 ; 3: 207-9

4 Klauser AG, Ting KH, Mangel E, Eibl-Eibesfeldt B, Müller-Lissner SA. Interobserver agreement in defecography. Dis Colon Rectum 1994; 37: 1310-6.

phy. Dis Colon Rectum 1994; 37: 1310-6.
5 Cummings JH, Jenkins DJA, Wiggins HS. Measurement of the mean transit time of dietary residue through the human gut. Gut 1976; 17: 210-8.

6 Arhan P, Devroede G, Jehannin B, Lanza M, Faverdin C, Arhan P, Devroede G, Jehannin B, Lanza M, Faverdin C, Dornic C, et al. Segment

7 Kuijpers HC, Bleijenberg G, de Morree H. The spastic pelvic floor syndrome. Large bowel outlet obstruction caused by pelvic floor dysfunction: a radiological study. Int $\mathcal{f} \mathrm{Colo}-$ rectal Dis 1986; 1: 44-8.
8 Barnes PR, Hawley PR, Preston DM, Lennard-Jones JE. Experience with posterior division of the puborectalis muscle in the management of chronic constipation. Brf Surg 1985; 72: 475-7.

9 Devroede G. Constipation. In: Sleisenger MH, Fordtran JS, eds. Gastrointestinal disease. Philadelphia: WB Saunders, 1994: 837-87.

10 Jones PN, Lubowski DZ, Swash M, Henry MM. Is paradoxical contraction of puborectalis muscle of functional importance? Dis Colon Rectum 1987; 30: 667-70.

11 Johanson JF, Sonnenberg A, Koch TR, McCarthy DJ. Association of constipation with neurologic diseases. Dig Association of constipation
Dis Sci 1992; 37: 179-86.

12 Bielefeldt K, Enck P, Zamboglou N, Moedder U, Erckenbrecht JF. Anorectal manometry and defecography in the diagnosis of faecal incontinence. $\mathcal{F}$ Clin Gastroenterol 1991; 13: $661-5$.

13 Duthie GS, Bartolo DCC, Miller R, Mortensen NJM, Virjee J. Anismus does not adversely affect the outcome of colectomy and ileorectal anastomosis for slow transit constipation. Gut 1989; 30: A735.

14 Infantino A, Masin A, Pianon P, Dodi G, Del Favero G, Pomerri F, et al. Role of proctography in severe constipation. Dis Colon Rectum 1990; 33: 707-12.

15 Enck P. Biofeedback training in disordered defecation. A critical review. Dig Dis Sci 1993; 38: 1953-60.

16 Klauser AG, Voderholzer WA, Heinrich CA, Schindlbeck NE, Müller-Lissner SA. Behavioral modification of colonic function. Can constipation be learned? Dig Dis Sci 1990; 35: $1271-5$.

17 Turnbull GK, Bartram CI, Lennard-Jones JE. Radiologic studies of rectal evacuation in adults with idiopathic constipation. Dis Colon Rectum 1988; 31: 190-7.

18 Halligan S, Bartram CI, Park HJ, Kamm MA. Proctographic features of anismus. Radiology 1995; 197: 679-82.

19 Andrews PJ, Barnes P, Borody TJ. Chronic constipation reversed by restoration of bowel flora. A case and a hypothesis. Eur f Gastroenterol Hepatol 1992; 4: 245-8. 